\title{
Validation of New Symptom-Based Fibromyalgia Criteria for Irritable Bowel Syndrome Co-morbidity Studies
}

\author{
Ami D Sperber, ${ }^{1,3,4,5 *}$ Smadar Akiva, ${ }^{3}$ Moshe Leshno, ${ }^{6}$ Zamir Halpern $^{5,7}$ and Dan Buskila ${ }^{2,3}$ \\ ${ }^{1}$ Department of Gastroenterology and ${ }^{2}$ Department of Medicine H, Soroka Medical Center, Beer-Sheva, Israel, ${ }^{3}$ Faculty of Health Sciences and \\ ${ }^{4}$ Sial Research Center for Family Medicine and Primary Care, Ben-Gurion University of the Negev, Beer-Sheva, Israel, ${ }^{5}$ Department of \\ Gastroenterology and Liver Diseases, Tel-Aviv Medical Center, Tel-Aviv, Israel, ${ }^{6}$ Faculty of Management and ${ }^{7}$ Sackler Faculty of Medicine, \\ Tel-Aviv University, Tel-Aviv, Istael
}

\section{Background/Aims}

There is significant co-morbidity between irritable bowel syndrome (IBS) and fibromyalgia syndrome (FMS). However, FMS is diagnosed by physical examination, which limits the conduct of co-morbidity studies in a large population-based study. The purpose of this study was to determine the diagnostic validity of a new symptom-based criteria in patients with FMS and/or IBS using the American College of Rheumatology (ACR) criteria as a gold standard.

\section{Methods}

The study participants consisted of women with FMS $(n=30)$, IBS $(n=27)$ and controls $(n=28)$. A new symptom-based diagnostic criteria for FMS comprised a regional pain scale and a visual analogue scale for fatigue. All subjects underwent a physical examination for FMS (ACR criteria) and structured questionnaires of regional pain scale and visual analogue scale for fatigue. A fibromyalgia intensity score was calculated and thresholds of tenderness were determined by a dolorimeter.

\section{Results}

The number of participants diagnosed with FMS in the entire study population $(n=85)$ was 31 by the new criteria. Compared to the $A C R$, the sensitivity of the new criteria was $82.9 \%$, specificity $96.0 \%$, positive predictive value $93.5 \%$ and negative predictive value $88.9 \%$. In addition, new criteria were useful for the diagnosis of FMS among the subjects with IBS. A fibromyalgia intensity score was significantly correlated with the threshold of tenderness $(r=-0.62, P<0.001)$.

\section{Conclusions}

The new symptom-based diagnostic criteria for the diagnosis of FMS can be used in large-scale clinical and epidemiological co-morbidity studies, in which physical examination is unfeasible. Gastroenterologists investigating the effects of co-morbid FMS in IBS patients can use these new ciriteria with confidence.

\section{(J Neurogastroenterol Motil 2011;17:67-72)}

Key Words

Comorbidity; Fibromyalgia; Irritable bowel syndrome; Physical examination

Received: June 24, 2010 Revised: November 3, 2010 Accepted: November 8, 2010

(c) This is an Open Access article distributed under the terms of the Creative Commons Attribution Non-Commercial License (http://creativecommons. org/licenses/by-nc/3.0) which permits unrestricted non-commercial use, distribution, and reproduction in any medium, provided the original work is properly cited.

*Correspondence: Ami D Sperber, MD, MSPH

Sial Research Center for Family Medicine and Primary Care, Faculty of Health Sciences, Ben-Gurion University of the Negev, Beer-Sheva, Israel 84101

Financial support: None.

Tel: +972-8-647-7433, Fax: +972-8-647-7623, E-mail: amy@bgu.ac.il

Conflicts of interest: None. 


\section{Introduction}

Irritable bowel syndrome (IBS) and fibromyalgia syndrome (FMS) are common functional disorders that share many characteristics. The prevalence of FMS among IBS patients ranges from $30 \%$ to as high as $70 \%$ in published studies. ${ }^{1-3}$ Our group reported that $32.4 \%$ of IBS patients met the American College of Rheumatology (ACR) diagnostic criteria for FMS and $32 \%$ of FMS patients met the Rome II diagnostic criteria for IBS. ${ }^{3} \mathrm{We}$ also found that patients with both disorders have more severe IBS symptoms, more psychopathology, lower health-related quality of life $^{4}$ and a lower score on the sense of coherence questionnaire for coping skills ${ }^{5}$ than patients with only one of these disorders. FMS is a soft tissue disorder characterized by diffuse musculoskeletal pain and specific tender points on physical examination. FMS is diagnosed in accordance with the ACR criteria of $1990,{ }^{6}$ namely widespread pain in combination with tenderness of 11 or more of the 18 specific tender point sites on physical examination.

Since many IBS and FMS patients have concomitant functional disorders in other body systems, eg, the chronic fatigue syndrome, the possibility of a common pathogenesis has been raised. $^{7-11}$ Large-scale clinical studies and epidemiological surveys could advance our understanding of these associations in terms of pathogenesis, the impact on symptom severity, healthrelated quality of life and patient care.

The diagnosis of IBS is symptom-based ${ }^{12}$ and is established mostly by the Rome criteria questionnaire. ${ }^{13}$ In contrast, the diagnosis of FMS requires physical examination by a trained examiner. This situation makes research into co-morbidity between FMS and other functional disorders, such as IBS, difficult, time consuming and inconvenient for the patient and the investigator. In effect, large-scale clinical or epidemiological studies of comorbidity between FMS and IBS are not feasible due to this limitation. Wolfe and Michaud ${ }^{14,15}$ developed and validated new criteria to diagnose FMS without requiring physical examination. The criteria are a composite score of their regional pain scale (RPS) and a visual analog scale (VAS) for chronic fatigue (for details see the Methods section below). In a validation study of 12,799 patients with rheumatic disease, Wolfe ${ }^{14}$ found a concordance rate of $73 \%$ between the ACR criteria and the new criteria. The authors concluded that the new criteria are useful for the diagnosis of FMS and have the advantage of not requiring physical examination.

The purpose of this study was to determine the test character- istics of the new diagnostic criteria in a population of IBS and FMS patients, as well as healthy controls, using the ACR criteria as the gold standard. The expectation is that the new criteria could be used in the future to facilitate studies of co-morbidity between IBS, FMS and other functional disorders.

\section{Materials and Methods}

\section{Study Groups}

The study population was comprised of women with FMS, women with IBS and female controls. The IBS patients and the controls were matched by age to the FMS patients. The women were recruited from the FMS and Gastroenterology clinics in the Soroka Medical Center, Beer-Sheva, Israel between January 2007 and March 2008. The FMS patients were recruited and tested first. IBS patients and controls were then matched by age $( \pm 3$ years) to the FMS group. The study group was comprised of women because this is a female-predominant disorder and we wanted to avoid potential confounders in this initial applicability study. The study was approved by the Helsinki committee (Internal Review Board) of the Soroka Medical Center. After receiving detailed information about the design of the study and its objectives and providing signed informed consent, each participant completed the survey questionnaire and underwent a physical examination to diagnose FMS, including palpation of tender points and determination of tenderness thresholds.

\section{Diagnostic Criteria}

\section{American College of Rheumatology criteria for fibromy- algia syndrome (1990)}

The ACR classification criteria of 1990 served as the gold standard. The ACR criteria are defined as:

Widespread pain has been present for at least 3 months. Pain is considered widespread when it is: (1) in both sides of the body and (2) above and below the waist. In addition, axial skeletal pain (cervical spine, anterior chest, thoracic spine or low back pain) must be present. Low back pain is considered lower segment pain. Finally, pain on digital palpation must be present in at least 11 of 18 defined tender point sites.

New composite score criteria for fibromyalgia syndrome

The diagnosis of FMS using the new criteria was based on a composite score of a survey questionnaire (RPS) and a VAS for fatigue, as follows:

Regional pain scale. This questionnaire is comprised of a 
list over 19 body areas. For each area the respondent was requested to grade the amount of pain or tenderness they felt in the specific area over the previous 7 days on a 4-item Likert scale of "none," "mild," "moderate" and "severe." Any response other than "none" was considered a positive response. Thus, the range of possible positive responses was $0-19$, as each of the 19 body areas could yield a positive or negative score. A total positive score for 8 or more areas was required for the diagnosis of FMS (see diagnosis of FMS, below). The RPS was translated into Hebrew and the translation was validated using the method published by one of the authors. ${ }^{16}$

Chronic fatigue visual analog scale. Participants were requested to mark the degree of fatigue they felt over the previous week on a VAS scale from $0-10$. A score of 6 or greater was required for the diagnosis of FMS.

\section{Composite score for the new diagnosis of fibromyalgia} syndrome

FMS was diagnosed if RPS $\geq 8$ and VAS $\geq 6$.

\section{Irritable bowel syndrome}

The diagnosis of IBS was based on the Rome II criteria, which were the latest update of the Rome criteria at the time the study was conducted.

\section{Study Protocol}

Each participant in the 3 study groups visited once in the study and the following variables were determined at that time: (1) number of tender points (of 18), by physical examination; (2) threshold of tenderness at tender points, by dolorimeter; and (3) FMS by RPS and chronic fatigue VAS.

The same investigator preformed all tender point and pressure threshold tests, described below, in all the study participants after being trained in the implementation of the tests by an expert rheumatologist with extensive experience.

\section{Tender Point Count}

The count of the 18 tender points was performed by thumb palpation at 9 symmetrical point sites. At first, manual pressure was demonstrated at a control site. Patients were told to expect a sensation of pressure, and to indicate if and when it became painful. Definite tenderness at any site was considered present if some involuntary verbal or facial expression of pain occurred, or a wince or withdrawal was observed. The amount of manual pressure over each tender point was about $4 \mathrm{~kg} / \mathrm{cm}^{2}$, as determined by a dolorimeter.

\section{Pressure Thresholds}

Pressure thresholds were assessed by a Chatillon dolorimeter (Chatillon Instruments, New York, NY, USA), a small instrument that has a maximum scale of $9 \mathrm{~kg}$, with a neoprene stopper as footplate and a diameter of $1.4 \mathrm{~cm},{ }^{17}$ at 13 sites ( 9 tender point sites and 4 control sites). The footplate was placed on a tender point and stabilized, if necessary, by the examiner's non-dominant hand to prevent (often painful) shifting of the footplate under pressure, while taking care not to add or subtract from the force applied. The dolorimeter was held close to the vertical position. Pressure was increased at a rate of about $1 \mathrm{~kg} / \mathrm{sec}$. The subject was asked to say "yes" when the sensation was no longer pressure, but became definite pain. Preliminary measures of control sites were done to familiarize the subject with the process and to discourage anticipation and/or exaggerated responses.

The intensity of FMS (FI) was calculated by means of the formula developed and validated by Wolfe et al (personal communication) as follows:

$$
\mathrm{FI}=(\mathrm{VAS}[\text { fatigue }]+[\mathrm{RPS} / 2]) / 2
$$

\section{Statistical Methods}

Sample size calculations determined that the required number of participants in each study group was 25 with $\alpha=0.05$ and $1-\beta=80 \%$. The required sample size to characterize the sensitivity of new criteria was calculated as described by $\mathrm{Li}$ and Fine, ${ }^{18}$ by testing $\mathrm{H}_{0}: \mathrm{Se}=\mathrm{Se}_{0}$ versus $\mathrm{H}_{1}: \mathrm{Se}=\mathrm{Se}_{1} \neq \mathrm{Se}_{0}$, where Se stands for sensitivity ( $\mathrm{Sp}$, for specificity, replaces $\mathrm{Se}$ in all calculations of specificity). By applying a standard normal approximation, we calculated the required number of patients with disease by using the following formula:

$$
n_{1}(\alpha, \beta)=\left(\frac{Z_{\alpha} \sqrt{S e_{0}\left(1-S e_{0}\right)}+Z_{\beta} \sqrt{S e_{1}\left(1-S e_{1}\right)}}{S e_{1}-S e_{0}}\right)^{2}
$$

where $n_{1}$ is the number of patients needed with $\alpha$ as the significance level, 1- $\beta$ is the power of the test and $Z_{\alpha}$ and $Z_{\beta}$ are upper $\alpha$ and $\beta$ quantiles of the standard normal distribution, respectively. The total population is $n_{1} / p$ where $p$ is the prevalence of the disease. In our case, assuming $\mathrm{Se}_{0}=0.7$ and $\mathrm{Se}_{1}=0.9$, the required sample size was 25 .

In addition to the cutoff points defined by Wolfe and Michaud $^{14,15}$ for the diagnosis of FMS with the new composite score, specificity and sensitivity rates were determined for a range 
of other cutoff points to see if any other cutoff point would generate better sensitivity and specificity than the pre-determined ones.

The Pearson's correlation coefficient was used to determine the correlation between the dolorimeter test of pain sensitivity (threshold) and the FI score from the survey questionnaire. Statistical significance was set at $P \leq 0.05$.

\section{Results}

The final study population was comprised of $30 \mathrm{FMS}$ patients, 27 IBS patients and 28 controls. The mean age $( \pm \mathrm{SD})$ was $51.7 \pm 12.9,53.1 \pm 12.4$ and $48.8 \pm 12.1$, respectively $(P$ $=0.41)$. Thirty-seven women in the 3 groups were diagnosed with FMS, 35 by the ACR criteria and 31 by RPS. Twenty-nine of these were diagnosed by both tests.

Table 1. Test Characteristics of Fibromyalgia Syndrome by Original Composite Score Compared to Gold Standard Test for Entire Study Population $(\mathrm{N}=85)$

\begin{tabular}{|c|c|c|c|c|}
\hline \multirow{2}{*}{\multicolumn{2}{|c|}{ Test }} & \multicolumn{2}{|c|}{ Composite score $^{\mathrm{a}}$} & \multirow{2}{*}{ Total } \\
\hline & & + & - & \\
\hline \multirow[t]{2}{*}{$\mathrm{ACR}$} & + & 29 & 6 & 35 \\
\hline & - & 2 & 48 & 50 \\
\hline Total & & 31 & 54 & 85 \\
\hline
\end{tabular}

${ }^{a}$ Composite score $=$ RPS $\geq 8$ and VAS $\geq 6$.

ACR, American College of Rheumatology; RPS, regional pain scale; VAS, visual analog scale.

Sensitivity $=82.9 \%$, specificity $=96.0 \%$, positive predictive value $=93.5 \%$, negative predictive value $=88.9 \%$.

Table 2. Test Characteristics of Fibromyalgia Syndrome by Modified Composite Score Compared to Gold Standard Test for Entire Study Population $(\mathrm{N}=85)$

\begin{tabular}{|c|c|c|c|c|}
\hline \multicolumn{2}{|c|}{ Test } & \multicolumn{2}{|c|}{ Composite score } & \multirow{2}{*}{ Total } \\
\hline & & + & - & \\
\hline \multirow[t]{2}{*}{$\mathrm{ACR}$} & + & 31 & 4 & 35 \\
\hline & - & 1 & 49 & 50 \\
\hline Total & & 32 & 53 & 85 \\
\hline
\end{tabular}

${ }^{\mathrm{a}}$ Composite score $=$ RPS $\geq 11$ and VAS $\geq 5$.

Abbreviations as in Table 1 .

Sensitivity $=88.6 \%$, specificity $=98.0 \%$, positive predictive value $=96.9 \%$, negative predictive value $=92.5 \%$.

\section{Test Characteristics (Sensitivity, Specificity and Positive and Negative Predictive Values)}

The sensitivity and specificity of the new composite diagnostic score for FMS were determined using the ACR criteria as the gold standard. These test characteristics were determined for the 85 women in the study population using the original cutoff points (composite score $=$ RPS $\geq 8$ and VAS $\geq 6$ ) (Table 1 ). The sensitivity and specificity were $82.9 \%$ and $96.0 \%$, respectively and the positive and negative predictive values were $93.5 \%$ and $88.9 \%$, respectively.

Several other cutoff points were also analyzed. The best test characteristics were found when the composite score for FMS was defined as RPS $\geq 11$ and VAS $\geq 5$ (Table 2). The sensitivity and specificity were $88.6 \%$ and $98.0 \%$, respectively, for the entire study population. The positive and negative predictive values were $96.9 \%$ and $92.5 \%$, respectively.

We did separate analyses in the subgroup of patients with IBS group $(n=27)$. The positive predictive value of the new

Table 3. Test Characteristics of Fibromyalgia Syndrome by Original Composite Score Compared to Gold Standard Test for $\mathrm{Pa}-$ tients With Irritable Bowel Syndrome $(\mathrm{n}=27)$

\begin{tabular}{|c|c|c|c|c|}
\hline \multirow{2}{*}{\multicolumn{2}{|c|}{ Test }} & \multicolumn{2}{|c|}{ Composite score $^{a}$} & \multirow{2}{*}{ Total } \\
\hline & & + & - & \\
\hline \multirow[t]{2}{*}{ ACR } & + & 3 & 2 & 5 \\
\hline & - & 0 & 22 & 22 \\
\hline Total & & 3 & 24 & 27 \\
\hline
\end{tabular}

${ }^{\mathrm{a}}$ Composite score $=$ RPS $\geq 8$ and VAS $\geq 6$.

Abbreviations as in Table 1.

Sensitivity $=60.0 \%$, specificity $=100.0 \%$, positive predictive value $=100.0 \%$, negative predictive value $=91.7 \%$.

Table 4. Test Characteristics of Fibromyalgia Syndrome by Modified Composite Score Compared to Gold Standard Test for $\mathrm{Pa}$ tients With Irritable Bowel Syndrome $(\mathrm{n}=27)$

\begin{tabular}{|c|c|c|c|c|}
\hline \multirow{2}{*}{\multicolumn{2}{|c|}{ Test }} & \multicolumn{2}{|c|}{ Composite score $^{a}$} & \multirow{2}{*}{ Total } \\
\hline & & + & - & \\
\hline \multirow[t]{2}{*}{$\mathrm{ACR}$} & + & 3 & 2 & 5 \\
\hline & - & 1 & 21 & 22 \\
\hline Total & & 4 & 23 & 27 \\
\hline
\end{tabular}

${ }^{\mathrm{a}}$ Composite score $=$ RPS $\geq 11$ and VAS $\geq 5$.

Abbreviations as in Table 1.

Sensitivity $=60.0 \%$, specificity $=95.5 \%$, positive predictive value $=75.0 \%$, negative predictive value $=91.3 \%$. 
composite score for FMS in IBS group was $100.0 \%$, negative predictive factor $91.7 \%$ with relatively low sensitivity (Tables 3 and 4).

\section{Association Between Pain Thresholds by Dolo- rimeter and the Fibromyalgia Intensity Score}

The dolorimeter determination of sensitivity threshold in the physical examination and the FI calculated on the basis of the RPS reflect a similar dimension. The mean FI was 8.4 for the FMS group and 3.8 for the IBS group. The Pearson's correlation coefficient between threshold of tenderness by dolorimeter and the FI was $-0.62(P<0.001)$. Thus, greater symptom intensity was inversely correlated with the threshold for pain and the correlation was statistically significant.

\section{Discussion}

The high rate of co-morbidity between functional disorders such as IBS and FMS and their shared characteristics have led some experts to propose that they share a common pathogenesis or even represent a single entity that has been split into separate diagnoses because of the hyper-specialization of the medical profession. ${ }^{19}$ Studies of the prevalence of co-morbidity between these 2 disorders and other functional disorders have the potential of increasing our understanding of each disorder and the links among them as well as contributing to the improvement of patient care.

However, co-morbidity studies that involve FMS are severely limited in scope by the need to perform a physical examination to determine the diagnosis on the basis of the ACR criteria of 1990. ${ }^{6}$ The conduct of large-scale clinical as well as epidemiological studies is unfeasible because of this limitation. In recognition of this problem, Wolfe ${ }^{14}$ developed the RPS as an alternative method to diagnose FMS in the research setting. Before using new study instruments (such as the RPS) and new diagnostic criteria (such as the composite score to diagnose FMS) in epidemiological and clinical studies, they must be validated by assessment of the test characteristics, in particular sensitivity, specificity and positive and negative predictive values, respectively.

The results of the present study, taken together with the validation studies published by Wolfe and Michaud, ${ }^{14,15}$ demonstrate that the RPS can be used as a valid substitute for the ACR criteria when large study groups make the physical examination unpractical or for epidemiological surveys that are conducted in large populations by mail, telephone or other indirect means.
Both the sensitivity and specificity calculated in this study ( $82.9 \%$ and $96.0 \%$, respectively) are acceptable. The higher specificity rate means that there are few false positives, while the somewhat lower sensitivity rates means that there could be some false negatives. Thus, studies of co-morbidity using these criteria could present a slight underestimate of the actual co-morbidity rates, which we believe is more acceptable than an overestimate. When analyzing other potential cutoff points, we calculated a sensitivity of $88.6 \%$ with a specificity of $98.0 \%$ using RPS $\geq 11$ and VAS $\geq 5$. This finding is of particular interest in that the number of painful regions by RPS ( $\geq 11$ ) corresponds with the minimum number of tender points required by the ACR at physical examination $(\geq 11)$. While we cannot recommend to use this cutoff point based solely on this finding, we do recommend the conduct of further validation studies that may show it to be a more useful cutoff for studies of co-morbidity between IBS and FMS in that it can provide us with a more accurate estimate of co-morbidity rates.

A limitation of this study is the restriction to female patients. This was done because IBS is a female-predominant disorder, as is fibromyalgia, and we wanted to have a homogeneous population to avoid potential confounders. This decision limits the generalizability of the results somewhat. Two other potential limitations of this study are that we did not ascertain the prevalence of IBS among FMS patients and that the propensity for reporting pain in IBS patients could lead to biased results.

In conclusion, gastroenterologists and other investigators interested in co-morbidity between IBS and FMS, as well as additional functional disorders and unexplained symptoms, can use the survey questionnaire (RPS). The new criteria can be used in large-scale general population-based studies and not only on studies of patient populations with IBS and other functional disorders. This is of interest because in each disorder there are many "non-patients" who are only identified through population-based surveys. For example, it might be used in a population survey looking into prevalence rates for different functional disorders from which we could learn about consultation behavior and differences between "consulters" and "non-consulters." The latter could not be done with the ACR criteria because of the need for physical examination. By enabling the conduct of large-scale clinical and epidemiological studies, our understanding would be broadened on the pathogenesis of co-morbid conditions and the implications for patients in terms of impact on symptom severity and health-related quality of life as well as potentially leading to improved patient care. 


\section{Acknowledgements}

The authors would like to express their gratitude to Dr. Frederick Wolfe who provided them with the RPS scale, and granted them permission to translate it into Hebrew and use it for this study. He also provided instructions for calculating the fibromyalgia intensity score on the basis of the RPS.

\section{References}

1. Triadafilopoulos G, Simms RW, Goldenberg DL. Bowel dysfunction in fibromyalgia syndrome. Dig Dis Sci 1991;36:59-64.

2. Sivri A, Cindas A, Dincer F, Sivri B. Bowel dysfunction and irritable bowel syndrome in fibromyalgia patients. Clin Rheumatol 1996;15: 283-286

3. Sperber AD, Atzmon Y, Neumann L, et al. Fibromyalgia in the irritable bowel syndrome: studies of prevalence and clinical implications. Am J Gastroenterol 1999;94:3541-3546.

4. Sperber AD, Carmel S, Atzmon Y, et al. Use of the functional bowel disorder severity index (FBDSI) in a study of patients with the irritable bowel syndrome and fibromyalgia. Am J Gastroenterol 2000;95: 995-998.

5. Sperber AD, Carmel S, Atzmon Y, et al. The sense of coherence index and the irritable bowel syndrome. A cross-sectional comparison among irritable bowel syndrome patients with and without coexisting fibromyalgia, irritable bowel syndrome non-patients, and controls. Scand J Gastroenterol 1999;34:259-263.

6. Wolfe F, Smythe HA, Yunus MB, et al. The American College of Rheumatology 1990 criteria for the classification of fibromyalgia. Report of the multicenter criteria committee. Arthritis Rheum 1990; 33:160-172.

7. Whitehead WE, Palsson O, Jones KR. Systematic review of the comorbidity of irritable bowel syndrome with other disorders: what are the causes and implications? Gastroenterology 2002;122:1140-1156.
8. Veale D, Kavanagh G, Fielding JF, Fitzgerald O. Primary fibromyalgia and the irritable bowel syndrome: different expressions of a common pathogenetic process. Br J Rheumatol 1991;30:220-222.

9. Hudson JI, Goldenberg DL, Pope HGJ, Keck PEJ, Schlesinger L. Comorbidity of fibromyalgia with medical and psychiatric disorders. Am J Med 1992;92:363-367.

10. Yunus MB. Fibromyalgia syndrome: clinical features and spectrum. J Musculoskelet Pain 1994;2:5-21.

11. Gruber AJ, Hudson JI, Pope HGJ. The management of treatmentresistant depression in disorders on the interface of psychiatry and medicine. Psychiatr Clin North Am 1996;19:351-369.

12. Appendix A. Diagnostic criteria for functional gastrointestinal disorders. In: Drossman DA, Corazziari E, Talley NJ, Thompson WG, Whitehead WE, eds. Rome II The Functional Gastrointestinal Disorders Diagnosis, Pathophysiology and Treatment: a Multinational Consensus. 2nd ed. McLean, VA: Degnon Associates 2000:569668.

13. Appendix B. Research diagnostic questions for functional gastrointestinal disorders. Rome II modular questionnaire: investigator and respondent forms. In: Drossman DA, Corazziari E, Talley NJ, Thompson WG, Whitehead WE, eds. Rome II the functional gastrointestinal disorders. 2nd ed. McLean, VA: Degnon Associates 2000:669-688.

14. Wolfe F. Pain extent and diagnosis: development and validation of the regional pain scale in 12,799 patients with rheumatic disease. J Rheumatol 2003;30:369-378.

15. Wolfe F, Michaud K. Severe rheumatoid arthritis (RA), worse outcomes, comorbid illness, and sociodemographic disadvantage characterize ra patients with fibromyalgia. J Rheumatol 2004;31:695-700.

16. Sperber AD, DeVellis RF, Boehlecke B. Cross Cult translation: Methodology and validation. J Cross Cult Psychol 1994;25:501-524.

17. McCarty DJ Jr, Gatter RA, Steele AD. A twenty pound dolorimeter for quantification of articular tenderness. Arthritis Rheum 1968;11: 696-697.

18. Li J, Fine J. On sample size for sensitivity and specificity in prospective diagnostic accuracy studies. Stat Med 2004;23:2537-2550.

19. Wessely S, Nimnuan C, Sharpe M. Functional somatic syndromes: One or many? Lancet 1999;354:936-939. 